W. S. KerR (G.B.). On this question of work ability, those of us who work in Britain may have had to fill in forms for the Department of Employment and Productivity, which used to be called the Ministry of Labour, and they have a remarkably useful, very small form called a D.P.I - a disabled person's number I form which shows for the Disablement Resettlement Officer a certain amount of work ability, can the patient push or pull, can he work sitting, can he walk, can he climb stairs and so on. This form is possibly something we could work from and elaborate.

Chairman. Summarising the discussion about this subject of classification we have heard some very interesting papers, and I should like to thank here in the first place Dr. Michaelis for starting the questionnaire on classification. He has great merit in this. I also would like to thank all other colleagues for their interesting contributions to this complex problem. I found the general discussion very useful and instructive although from all the papers and the ensuing discussions some of you might think we are now more confused than ever. However, being an optimist, I hope we will sort out the confusion in due course.

Dr. Parsch has given very detailed views about classification of a special subject of spina bifida and we look forward to the more concise classification from our friends in Heidelberg. Professor Jochheim has now proposed the setting up of a panel to try to co-ordinate the various views expressed, in particular the discrepancies between the French and what is called Anglo-Saxon views on classification. You may remember that when we discussed the matter first that I made the suggestion to set up such a co-ordinating committee, and I hope that will be done in the near future. Thank you again for your excellent contributions.

\title{
AN OUTLINE OF RECENT WORK ON THE SPINAL CORD OF THE CAT
}

\author{
By Professor C. G. Phillips, F.R.S., F.R.C.P. \\ University Laboratory of Physiology, Oxford
}

IN introducing this Session on Spinal Shock, I shall try to give as briefly as I can, and illustrate by a very few of the leading experiments some glimpse of today's neurophysiological picture of the lumbosacral spinal cord of the cat. Because the Society's interests are centred on the physiology of the spinal segments after their partial or total disconnexion from the brain, I shall draw largely on the work of Professor Anders Lundberg and his colleagues, first in Lund and now in Gothenburg. They have been studying the suprasegmental control of reflex arcs and investigating the remarkable differences between the patterns of segmental reflex activity seen in the cords of 'decerebrate' preparations on the one hand and 'spinal' preparations on the other. From such a large volume of relevant work, any selection cannot help but be arbitrary, and in trying to present such a selection succinctly, some degree of dogmatism seems inescapable. Therein lie great dangers of creating, in the minds of readers and audiences, undesirable vested interests in the details of an oversimplified story. One's hope is rather to encourage those responsible for the medical care of patients with spinal injuries to make and maintain contact with an exciting and enlarging field of experimental work.

The spinal cord (fig. I, S) is the nerve-centre of trunk and limbs. It contains 
the nervous apparatus whereby stimulation of the receptive fields of their skin (fig. I, aa') evokes simple reflex movements (Sherrington's 'exteroceptive reflexes' fig. I, $\mathrm{bb}^{\prime}$ ); and for elaborating certain stereotyped patterns of movement, e.g. the alternating flexion and extension phases of stepping; scratching; copulatory move-

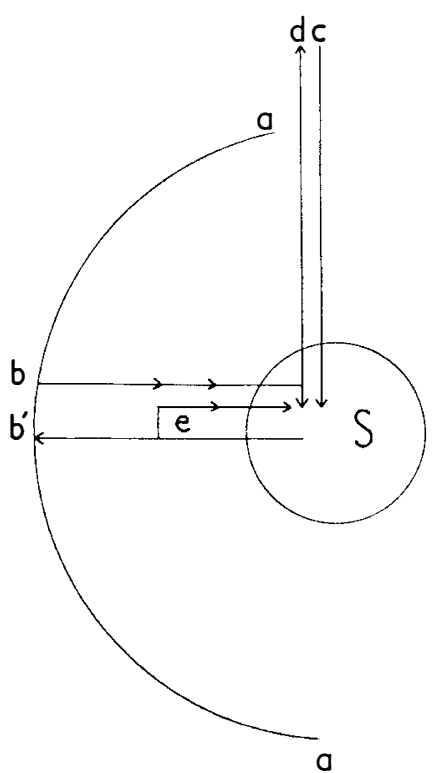

FIG. I ments. But as one ascends the evolutionary ladder, one finds this spinal apparatus becoming more and more dependent upon control by the brain (fig. I, c), and, through the brain, by the vestibular and visual receptive fields. Thus it comes about that when the cord is disconnected from the brain, the initial depression of reflex activity, or 'spinal

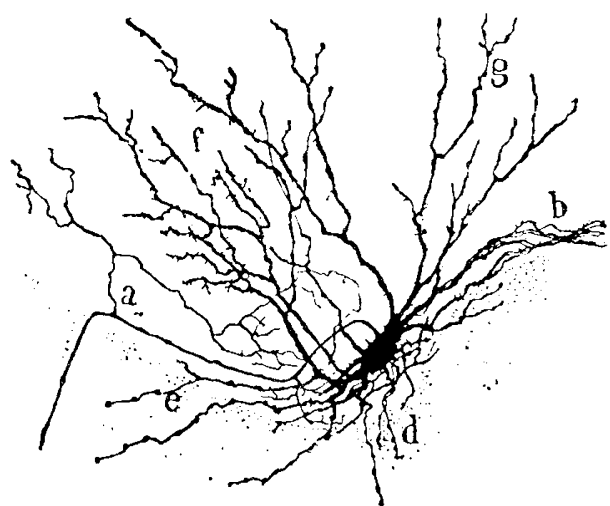

FIG. 2

Fig. I.-S, Spinal cord. aa', Skin receptive fields. bb', Exteroceptive reflexes. C, Brain control descending to $S$. d, Information from receptive fields ascending to brain. e, Circuits governing both reflex and brain-controlled movements ('proprioceptive reflexes').

Fig. 2.-A spinal motoneurone (Ramon y Cajal). a, Recurrent collateral branches of axon. b, d, e, f, g, Dendrites covered with synapses. The soma (near d) could be nearly Ioo $\mu$ long.

shock', is more severe and prolonged in man than in the lower vertebrates. Even in the cat, the spinal reflexes are powerfully controlled by the brain: they can be exaggerated or suppressed by nerve paths descending from the brain, their receptive fields being correspondingly widened or narrowed in area. When wasps are about, one is sometimes surprised by the vigour of one's reflex withdrawal from some unexpected light touch.

But the skin is not only for the initiation of reflexes: its receptive fields form part of the general interface between the animal and the world outside, and keep the brain informed of changes in the immediate environment (fig. I, d). On the basis of this information and that from the vestibular, visual, auditory and olfactory fields, and under pressure of internal 'drives', the brain projects upon the spinal reflex apparatus an ever-shifting pattern of posture and movement, for transcending in range and complexity the relatively simple patterns that can be evoked by segmental stimulation.

Much of the reflex apparatus is concerned with the internal governing of 
position and movement (Sherrington's 'proprioceptive reflexes'), and it is of this part that our knowledge is most detailed and complete. The receptive fields are internal (in muscles, tendons, joints). These arcs are represented by e in Figure $\mathrm{I}$. Their operation is common to reflexly-induced (Figure I, b) and brain-evoked (Figure I, c) activities. They are outlined in Figure 4, and will be discussed in greater detail below.

Fundamental Properties of Motoneurones and Reflex Arcs. For all these activities the ventral horn cells (spinal motoneurones, Figure 2) with their branching axons and the cluster of muscle fibres innervated by each (the 'motor units') constitute, in Sherrington's terminology, the 'final common path'. The force developed in any movement depends on the number of motoneurones that are recruited into action, and on the frequency of the impulses they discharge.

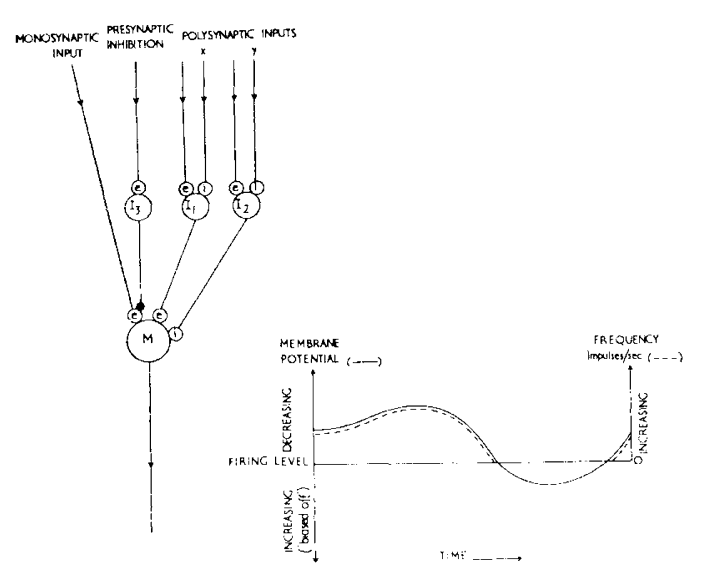

FIG. 3

Inputs to a spinal motoneurone. The elaborately tapering and ramifying presynaptic arborizations are here represented as circles. e, Excitatory synapses. I, Inhibitory synapses. $M$, Motoneurone (see fig. 2). $\mathrm{I}_{1}, \mathrm{I}_{2}, \mathrm{I}_{3}$, Interneurones.

Bottom right: Full line shows imaginary fluctuations of the membrane potential of the motoneurone in response to shifting interplay of excitatory and inhibitory synaptic actions upon its membrane. Dotted line shows the resulting changes in the frequency of the impulses sent by the motoneurone to the muscle fibres of its motor unit.

The soma and dendrites of the motoneurones are covered by synapses-areas of contact between the motoneurone membrane and the membranes of the terminal arborisation of afferent axons: some of these are primary afferent axons entering the cord by the dorsal roots, others are axons lying wholly within the cord and brain. On the arrival of presynaptic impulses these terminals secrete chemical transmitters across the narrow gaps that separate the pre- and post-synaptic membranes. The excitatory synapses diminish, the inhibitory synapses increase the electrical negativity of the interior of the motoneurone (Eccles, 1964). The experiments of Granit (I966A) and his colleagues in Stockholm have shown how accurately the opposing effects of these excitatory and inhibitory inputs on the membrane potential are algebraically summed to determine the frequency of impulse discharged by the motoneurone ( $M$, fig. 3) to the muscle fibres of its motor unit, in the manner conceived by Sherrington long ago. Each excitatory imput adds its quota of impulses-per-second to the output. Each inhibitory imput subtracts its quota. When the motoneurone is silent, it is not merely switched off, but 'biased off' (figure 3) so that its sensitivity to incoming excitatory imputs can be varied. 
These excitatory and inhibitory inputs to the motoneurone membrane are subject to controls operating upstream of the final common path (fig. 3). Experimental evidence for some of the following statements will be given below. The quantity of transmitter liberated by each impulse arriving at a presynaptic arborisation is not fixed, but depends on the prevailing electrical potential across the presynaptic membrane. Following an increase in impulse traffic this potential increases: each impulse then liberates a larger quantity of transmitter and exerts an increased synaptic action on the motoneurone ('post-activation potentiation'). Pre-synaptic arborisations can have their membrane potential reduced by the activity of interneurones ( $\mathrm{I}_{3}$, fig. 3 ). Presynaptic impulses then liberate less transmitter ('presynaptic inhibition'). If a motoneurone is being subjected to a steady excitatory synaptic bombardment by interneurones $\left(\mathrm{I}_{1}\right.$, fig. 3 ), and these are inhibited (x, fig. 3), the responsiveness of the motoneurone to its other inputs will be decreased ('disfacilitation'). If inhibitory interneurones ( $\mathbf{I}_{2}$, fig. 3 ) are themselves inhibited (y, fig. 3), the motoneurones's responsiveness to other inputs will be increased ('disinhibition').

Experimental Methods. The operation of these structures and processes in reflex activity is now being worked out in considerable detail, and before looking at a sample of the experimental results, something needs to be said about the methods that will be illustrated in Figures 5, 6, 7, 9 and 10.

It is usual to send afferent impulses into the cord by electrical stimulation of peripheral nerves rather than by natural stimulation of receptive fields. This has the advantage that timing can be exact, but raises difficulties about the correspondence between the artificially evoked inputs and particular receptive fields. Much of the necessary discrimination can be achieved by separately stimulating the nerve branches from skin, muscles and joints. Within these groups some further discrimination can be achieved by grading the strength of the electrical stimuli. For example, the different groups of afferent fibres in a cat's muscle nerves (fig. 4) differ in diameter: those from the 'primary' receptors of the muscle spindles ('Group Ia') are the thickest, those from the Golgi tendon organs ('Group Ib') are next in size, and those from the 'secondary' spindle receptors ('Group II') are thinner still. The thinnest ('Group III') are from pain endings, etc. A single weak stimulus to a muscle nerve will therefore start a volley of impulses in Group Ia fibres only. Graded strengthening of the stimulus will add, first, Group Ib, then Group II, and finally Group III to the volley. Since the Group I impulses travel most rapidly, they reach the cord first; the Group II and III components of the volley lag behind and enter the cord later. The discrimination is always checked in records from the dorsal root entry zone. It is not absolute, and there remains always some possible uncertainty about the degree of functional overlap between the different groups. For the convenience and precision of electrical stimulation some price will probably always have to be paid. For example, different axons in a joint nerve signal different ranges of the angular displacement of the joint. Such discrimination is inevitably lost when all are stimulated to discharge a synchronous volley. But these are problems for the future; the method has made possible very rapid progress in tracing the main reflex circuits in the lumbosacral segments of the cat.

The responses of the motoneurones to these inputs can now be studied with intracellular microelectrodes (Eccles, 1964). A cell can be identified as a moto- 
neurone by the impulse that travels backwards into it when its axion is stimulated antidromically in the ventral root or muscle nerve. The excitatory and inhibitory synaptic actions generated by afferent impulses are directly recorded as waves of decreased or increased internal negativity of the cell. These waves are called, respectively, excitatory post-synaptic potentials (EPSP) and inhibitory postsynaptic potentials (IPSP). EPSPs will be illustrated in Figure 6, Figure 9, c, and Figure IO, and an IPSP in Figure 9, F.

Complementary to such detailed investigations of sampled neurones are studies of the reflex responsiveness of whole pools of motoneurones to the particular inputs one is interested in. Such studies will be illustrated in Figures 5 and 7. They take advantage of the fact that the motoneurones of the pool belonging to a particular muscle receive a monosynaptic excitatory input (Figure 3 ) from the Group Ia axons from that muscle (Figure 4). This is the system the clinician uses each time he elicits a 'tendon jerk': the tendon-tap briefly lengthens the muscle, and sends in a Group Ia volley, which synaptically excites some of the motoneurones to fire a fairly synchronous volley down the motor nerve. The same system is used by the clinical neurophysiologist each time he elicits an H-reflex (Hoffmann refiex) by stimulating the human popliteal nerve with shocks below threshold for its motor axons but above threshold for its Group Ia axons-the method discovered by Paul Hoffmann in 1922. The experimental physiologist also uses the monosynaptic test reflex as a tool for investigating the physiological state of a motoneurone pool. He records the pool's reflex response to a standard-sized Group Ia input by measuring the size of the resulting motor volley. This size gives a measure of the number of motoneurones that have actually fired a single impulse: many others in the pool will have been subliminally excited (Sherrington's 'subliminal fringe'). He is now ready to investigate whether another input to the pool is excitatory or inhibitory. He fires in the unknown and follows it by the standardised Group Ia volley. If the unknown was inhibitory, it will prevent some of the motoneurones from responding in the test reflex, and that reflex will be smaller than the control reflex (compare fig. 5, C and D). If on the other hand the unknown was excitatory, some cells of the subliminal fringe will be brought to firing level, and the test reflex will be larger than the control (compare fig. 5, E and F).

The onset, waxing and waning of these excitatory or inhibitory synaptic actions of 'unknown' inputs can be plotted as a graph, as in Figure 7. The 'unknown' input goes in at time 0 . The testing Group Ia volley goes in at different times thereafter, and the size of the monosynaptic reflex response of the motoneurone pool is plotted as a percentage of the control (IOo per cent.; note horizontal line in Figure 7). Such curves beautifully show the onset and time-course of excitation or inhibition.

Much of the usefulness of such investigations of single motoneurones and motoneurone pools stems from their ability to detect events occurring at neural levels lying upstream of the final common path (fig. 3).

Post-activation potentiation of transmission from presynaptic arborisations has already been mentioned. After prolonged high-frequency stimulation of Group Ia afferents, the size of the monosynaptic test reflex elicited by the same afferents is found to be much increased. The test reflex does not return to normal for many minutes. The monosynaptic reflex response of the motoneurone pool to a testing volley in Group Ia afferents other than those subjected to the conditioning high-frequency stimulation is unchanged. Therefore the effect is presynaptic, and 
confined to the 'conditioned' presynaptic arborisations. This knowledge could be important to the physician who wishes to encourage the earliest possible return of extensor reflexes-recovery which could be delayed if the synapses lay long in disuse.

Presynaptic inhibition was discovered in intracellular experiments on single motoneurones. Certain inputs, which by themselves produced no detectable change in the motoneurone membrane, would reduce the size of a testing monosynaptic EPSP. The effect can be shown to be due to depolarisation of the presynaptic arborisations of the testing input by three methods. Intracellular recording from their afferent axons reveals the depolarisation of the individual presynaptic membranes. Extracellular recording detects an electric wave, generated by the depolarisation of the massed arborisations. And electrical stimulation of the arborisations shows that their excitability waxes and wanes with the waxing and waning of the massed electrical wave.

The presence of interneurones in a reflex arc can be readily established. It is characteristic of such 'polysynaptic' arcs that a small input, recorded from the primary afferent axons at the dorsal root entry zone, produces no effect on the membranes of the motoneurones. This proves that these primary afferent axons do not form synapses on the motoneurones. Exploration with microelectrodes discovers neurones, lying more dorsally in the grey matter, which do receive synaptic actions from these primary afferent axons. Such neurones are not motoneurones, for stimulation of ventral roots or muscle nerves sends no antidromatic impulses into them. When the primary afferent input is stepped up, or another input is added (e.g. corticospinal: fig. 9) the interneurones are brought to firing level. Only then do the membranes of the motoneurones show EPSPs or IPSPs. These are delayed in relation to monosynaptic EPSPs (fig. 6, P). The larger the number of interneurones interposed between the primary afferent arborisations and the motoneurones in a polysynaptic arc, the longer the delay.

Proprioceptive Regulation of Movement and Posture. We are now ready to look at the segmental circuits which govern the output of the motoneurones and which are common to reflexly evoked and brain-evoked movements and postures (fig. I, e). These circuits are presented in crude outline in Figure 4.

Muscle-groups are linked mechanically at joints, and the diagram represents this situation by showing a group of flexors and a group of extensors attached to the two ends of a see-saw whose bearing represents a joint. The assemblies of motoneurones innervating the two muscle-groups are indicated by the large filled circles of Figure 4. The flexors cannot shorten without lengthening the extensors, and vice versa. As has been stated already, the joint capsule contains receptive fields which supply information of angular movement and position. The larger axons in articular nerves have variable reflex effects, the smaller evoke the pattern of generalised flexion of the limb. The diagram is silent on the intraspinal destination of the joint afferents, shown as arising from the hinge of the see-saw.

When muscle contracts against resistance, its motoneurones come under disynaptic inhibition from tension receptors in the tendons. This is drawn, for the flexor group, at the right of the diagram (Ib). The corresponding circuit for the extensor group is omitted to avoid complicating the diagram.

When muscle is passively lengthened, as by gravity or by contraction of an antagonistic group, two classes of receptive field are excited within it. One, 
'secondary' endings of the muscle spindles, attached to Group II axons (II, fig. 4), signals length at any instant. This is illustrated by the dotted line below the Group II input from the extensor muscle group. It is a graph of impulse frequency (vertical axis) against time (horizontal axis). The stimulus was a lengthening of the muscle at constant velocity. The lower horizontal line shows the impulse frequency, steady at the shorter length. The sloping line silows the linear increase in frequency as the muscle is lengthened. The upper horizontal line shows the final frequency, steady at the greater length. The central connexions and effects of the Group II input are still being worked out, and are therefore left blank in the diagram. It is not connected monosynaptically to the motoneurones, so its actions must be polysynaptic. Very recent work by P. B. C. Matthews strongly suggests that it contributes to the reflex increase in the motoneurone discharge when an experimentally spastic extensor muscle is stretched. When stimulated electrically, the Group II input from any muscle evokes the generalized flexion pattern of the limb.

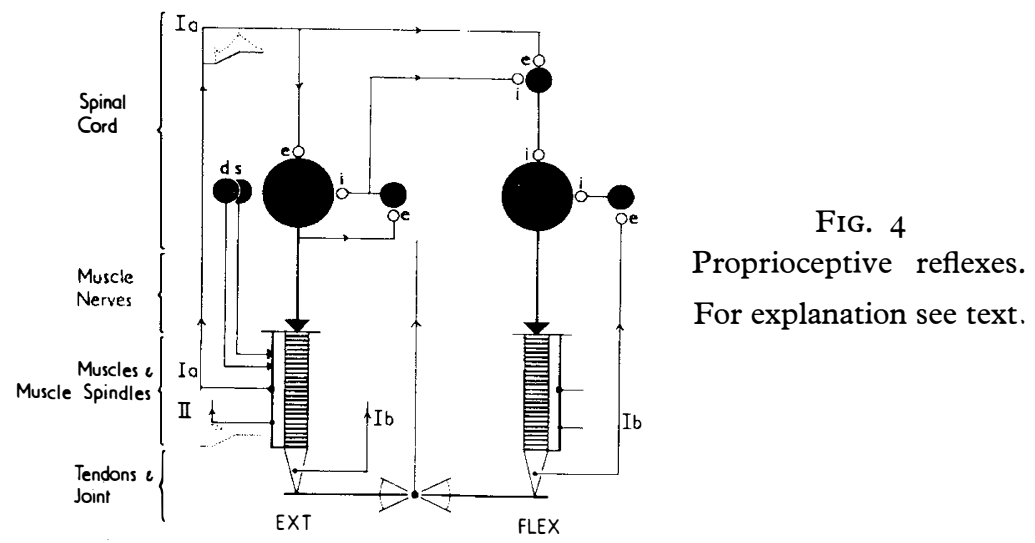

The other class of muscular receptive field is represented by the 'primary' endings of the muscle spindles, attached to Group IA axons (fig. 4, Ia), which supplies the central nervous system with information of length when the muscle is stationary, but also of velocity when the muscle is being lengthened. A typical graph of impulse frequency against time is shown by the dotted line in the inset at top left of Figure 4. The lengthening is indicated by the full line. The faster the muscle is lengthened, the higher the frequency. Enough has been said already about the monosynaptic connexion of the Group Ia input to the motoneurones of its own muscle; it remains only to add the disynaptic inhibition of the motoneurones of its antagonist (fig. 4, top right). The corresponding central connexions made by the Group Ia input from the flexors are omitted to avoid confusing the diagram (but are shown in fig. II). These reciprocal connexions between antagonistic groups are the most elemental of the arrangements that are the basis of Sherrington's 'reciprocal innervation'-relaxation of antagonists, when appropriate, when prime movers contract. Polysynaptic connexions are also made by the Group Ia input, but little is known about these, and the diagram ignores them.

We come now to the fusimotor neurones or ' $\gamma$ motoneurones' (fig. 4, d, s) which innervate the intrafusal muscle fibres of the muscle spindles. They enable 
the central nervous system to adjust the sensitivity of the 'primary' and 'secondary' receptors. The fusimotor neurones fall into two classes. One class, the static fusimotor ( $\left.{ }^{\prime} \gamma_{s}^{\prime}\right)$, increase the impulse-frequency of primary and of secondary receptors when the muscle is stationary. The other class, the dynamic fusimotors $\left({ }^{\prime} \gamma_{\mathrm{D}}{ }^{\prime}\right)$, increase the impulse-frequency of primary receptors when the muscle is being lengthened. It is obvious that such increases will increase the central synaptic potency of the spindle inputs. The 'adjusted' input frequencies can be as high as those which produced post-activation potentiation in the experiments referred to earlier. In the experimental spasticity of a Sherringtonian decerebrate, there is abundant evidence of increased fusimotor activity, but the increased monosynaptic.Group Ia input cannot alone account for the sustained discharge of the extensor motoneurones: other sources of motoneurone excitation (e.g. vestibulospinal-see below) are certainly at work.

In the absence of fusimotor activity, active shortening of the muscles 'unloads' the spindle receptors, and therefore silences their discharge; for the spindles (as fig. 4 illustrates) lie 'in parallel' with the muscle. But suppose that a reflex 'command' (fig. I, b) or brain 'command' (fig. I, c) activates the (' $\alpha$ ') motoneurones and fusimotor ( $\gamma$ )' neurones together. This is what Granit calls ' $\alpha \gamma$ linkage'. Then the Group Ia input need not be silenced, but could continuously reinforce the 'command' at the motoneurone membrane, and continuously inhibit the motoneurones of the antagonistic muscles. If the movement were resisted by an external load, the unloading of the spindles would be checked: their discharge would therefore increase (without any change in the fusimotor activity) and give increased support to the 'command' at the motoneurone membrane. Such a servomechanism would tend to compensate for changes in external load.

The last of the control circuits shown in Figure 4 is the disynaptic inhibitory feedback from collaterals of motor axons (cf. fig. 2, a). To keep the diagram simple this has been drawn for extensor motoneurones only, but the flexors have it also. It tends to limit the output frequency. Very recent work in Gothenburg (Hultborn, Jankowska \& Lindström, I968) has discovered that the inhibition is directed also to the inhibitory interneurones of the Group Ia pathway to the antagonistic motoneurones. This possibility of switching off one of the mechanisms of reciprocal inhibition might be important when co-contraction of antagonists is required.

Exteroceptive Reflexes. It is helpful to view the exteroceptive reflexes (fig. I, $b^{\prime}$ ') as grafted on to the 'proprioceptive' systems of Figure 4, engaging the final common path and its control circuits as a functional whole.

The stereotyped pattern of generalized flexion of a limb is to be seen not only in response to noxious stimulation of the skin, but also as the flexion phase of reflex stepping. The nociceptive flexion reflex is polysynaptic. It engaged the flexor motoneurones and the flexor fusimotoneurones together in $\alpha \gamma$ linkage. The extension of the opposite limb (Sherrington's 'crossed extensor reflex') and of the forelimbs involves long chains of interneurones and deserves re-investigation by modern methods. The same may be said of the reflex extension of a limb that may be evoked by gentle pressure on the footpads or by gently stroking the skin of the groin. Hagbarth found that pinching the skin overlying a muscle caused reflex contraction of that muscle: if it was an extensor muscle, there was a local distortion of the generalised flexor pattern. Of the complex nervous machinery of scratching and reflex locomotion little is yet known, but the effects in this con- 
nexion of DOPA (L3,4-dihydroxyphenylalanine-see below) on the spinal cord are of great interest.

Brain Control of Segmental Reflexes. Although stereotyped, the segmental reflexes are not deterministic in the sense that the same stimulus applied to a receptive field must always elicit an identical reflex action. Such determinism is most nearly approached when the spinal cord has been disconnected from the brain. In normal circumstances the segmental reflex arcs are subject to opening and closure by the brain. The brain can open a reflex arc by adding excitation and withdrawing inhibition, and can close it by adding inhibition and withdrawing excitation. Professor Lundberg and his colleagues have placed this subject in the forefront of modern work on the physiology of spinal reflexes, and although its details relate to the cat and not to man, it raises important principles for the clinician who is responsible for patients with spinal injuries.

The investigation begins as an analysis of the differences between the reflex behaviour of intact anaesthetised cats on the one hand and unanaesthetised decerebrate and spinal cats on the other. In the decerebrate the extensor reflexes are exaggerated and the flexor reflexes diminished. The natural balance between extensor and flexor reflexes has been upset by differentially disconnecting their arcs from the normal tonic excitatory and inhibitory controls from the brain. The classical view of the exaggerated extensor reflexes is that they have been released from tonic inhibitory control. What of the diminished flexor reflexes? Is their depression due to withdrawal of tonic excitatory control, the state classically known as diaschisis or 'shock'? If so, one would expect them to remain depressed when the spinal cord was cut. But their depression might instead be due to preponderance of tonic inhibition from the hindbrain. If so, cutting the cord should release them from this inhibition. This is what is found experimentally.

The next stage is to identify the descending pathways and test them for excitatory and inhibitory effects on flexor and extensor arcs. This is done in two ways: first, by making successive partial lesions of the brainstem and cord in those parts of their cross-section areas known to be occupied by specific descending tracts, and testing the reflex arcs by the electrophysiological methods already described in order to discover which of the successive lesions causes the loss of the characteristic tonic excitatory or inhibitory control. Second, the descending tracts or their cells of origin in the brain have been stimulated electrically, and their excitatory or inhibitory effects detected by the same electrophysiological investigations of the primary afferent axons, interneurones, fusimotor neurones and motoneurones of the flexor and extensor reflex arcs. From these refined experiments, some of which will now be illustrated, a picture of the brain's control of the segmental reflexes is being built up. The picture leads towards the concept of different states of imbalance between specific controls and away from such global concepts as 'spinal release' or 'spinal shock'. Loss of tonic inhibition may be preponderant after some disconnexions, loss of tonic excitation after others, but each state of imbalance needs to be understood in detail, and ultimately related to the overall neurological situation (loss of vestibular control, visual control, etc.).

Experiments on the Tonic Effects on Reflex Arcs of Transection of the Hindbrain at Different Levels. Figure 5 shows the changes in responsiveness of a pool of flexor motoneurones to pinching the skin of the leg, brought about by successively caudal transections of the neuraxis. The responsiveness was tested by the monosynaptic 
method. In the decerebrate, pinching the skin of any part of the leg or thigh had almost no effect on the monosynaptic excitability of the pool. This can be seen by comparing the monosynaptic reflex of Figure 5, B with the control monosynaptic reflex of Figure 5, A. Evidently, the excitation from the skin was not getting through to the flexor motoneurones, and was presumably blocked at the interneuronal level by tonic descending inhibition. The neuraxis was then transected at pontine level. Pinching over a wide area of skin now depressed the excitability of the pool (compare D with its control, C; note minus signs on diagram of leg). Not only was cutaneous excitation still failing to get through to the motoneurones: the fresh lesion had released from tonic restraint some other path by which cutaneous impulses may inhibit flexor motoneurones. Finally, the spinal cord was cut. Pinching, over most of the same skin area, now increased the excitability of the pool (compare F with its control, E; note plus signs on diagram). The tonic descending

FIG. 5

Changes in responsiveness of a flexor motoneurone pool to pinching the skin of different parts of the leg and thigh, caused by successively caudal transections of the CNS, and tested by the monosynaptic method. For explanation see text. (Holmqvist \& Lundberg, I96I.)

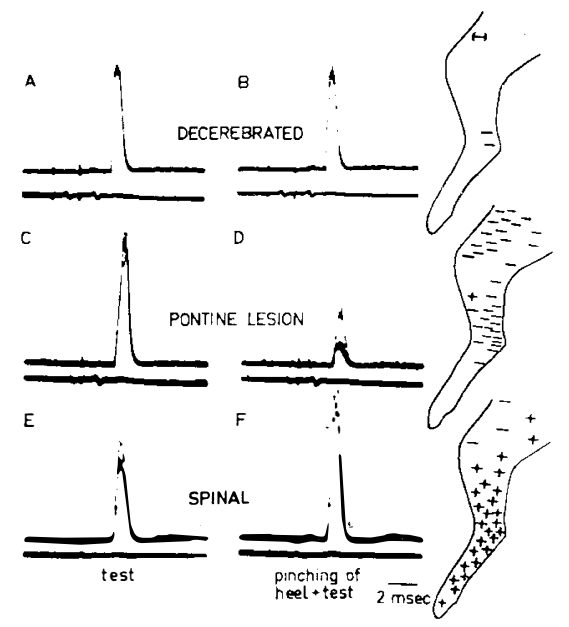

inhibition of the flexor interneurones had at last been removed, and pinching now had a strong excitatory action on the flexor motoneurones, as revealed by the monosynaptic test.

This abolition of flexion reflexes by decerebration and their restoration by cutting the spinal cord can also be revealed by intracellular experiments on single flexor motoneurones (fig. 6). Records A-H are typical of flexor motoneurones seen in the decerebrate state. As the strength of shocks to the muscle nerve is increased from $\mathrm{I} \cdot 07$ to $\mathrm{I} \cdot 34$ times threshold, the monosynaptic EPSP reaches its maximal size, and further increase in strength adds no further synaptic excitation. Records L-P are typical of flexor motoneurones in the acutely spinal state. Compare, first, record $\mathrm{P}$ (spinal) with record $\mathrm{H}$ (decerebrate). The monosynaptic EPSP in record $\mathrm{P}$ has a polysynaptic EPSP superimposed on its declining tail. This new EPSP starts 3.5 msec. later than the monosynaptic EPSP (see arrow). Observe its first appearance in record $\mathrm{L}$, and its growth in records $\mathrm{M}$ to $\mathrm{P}$ with increasing stimulation of the nerve. This 'flexor reflex' action on the motoneurone membrane is due to the firing of 'flexor' interneurones, released by spinal section from the tonic inhibition prevailing in the decerebrate state.

It is of great interest that the static fusimotor neurones are silent and unresponsive in the acutely spinal cat. Jansen has shown with his colleagues in Oslo 
that only the dynamic fusimotors are spontaneously active and responsive to stimulation. The former finding helps to explain the loss of well-sustained extensor reflexes in the acutely spinal cat: the latter finding helps to explain the survival of the knee jerk.

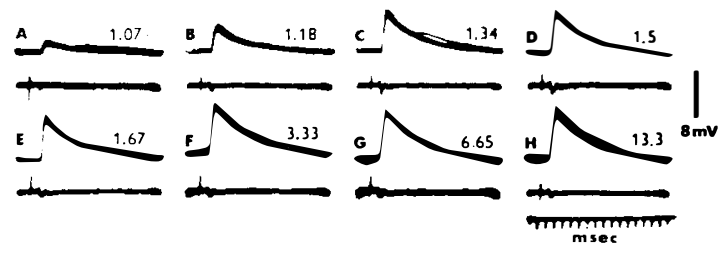

FIG. 6

A-H, Responses of a flexor motoneurone to increasingly-strong stimuli to the nerve to its muscle. Decerebrate cat. Note uncomplicated decay of the monosynaptic EPSP, even in $\mathrm{H}$, when the stimuli (1 $3.3 \times$ threshold strength) would have ex-

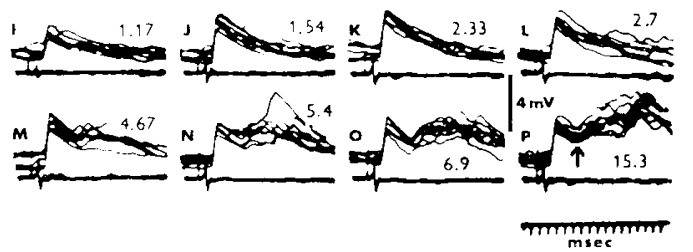
cited flexion reflex afferents. I-P, Acute spinal cat. Another flexor motoneurone. Compare $\mathrm{M}, \mathrm{N}, \mathrm{O}, \mathrm{P}$ with E, F, G, H. The decay of monosynaptic EPSP is now interrupted by the polysynaptic EPSP elicited by flexion reflex afferents. (Lundberg, 1964).

Effects of Incomplete Lesions of the Spinal Cord. Figure 7 illustrates the use of successive partial lesions of the cord in trying to discover the brain paths that normally keep reflex arcs under tonic control. The effect of a flexor reflex input on a flexor motoneurone pool was plotted by monosynaptic testing. At the outset the preparation was decerebrate. The flexor reflex input had no effect on the response of the motoneurone pool to the monosynaptic test (horizontal line, Ioo per cent.) $j$ the flexor interneurones were tonically inhibited. Cutting the dorsal columns (see dot against upper inset diagram) failed to remove this inhibition (dots, corresponding to upper inset diagram, fall on the Ioo per cent. line).

FIG. 7

Effects of successive cord lesions on a flexion reflex arc in a decerebrate cat. For explanation see text. (Holmqvist \& Lundberg, 1959.)

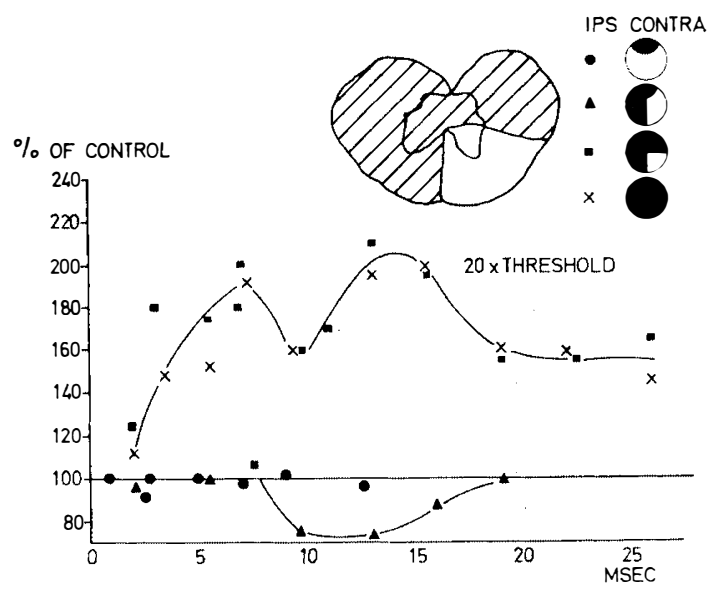

Ipsilateral hemisection of the cord still failed to remove the tonic inhibition: the flexor input now inhibited the flexor pool (fig. 7, triangles; compare effect of pontine lesion, fig. 5). Section of the remaining (contralateral) dorsal quadrant of the cord did finally release the flexor interneurones: the flexor reflex input now 
produced a large increase in motoneuronal excitability (fig. 7, squares). Completing the transection of the cord had no further effect (fig. 7, crosses). The descending pathway therefore travelled in the dorsal quadrant. It is separate from the pathway

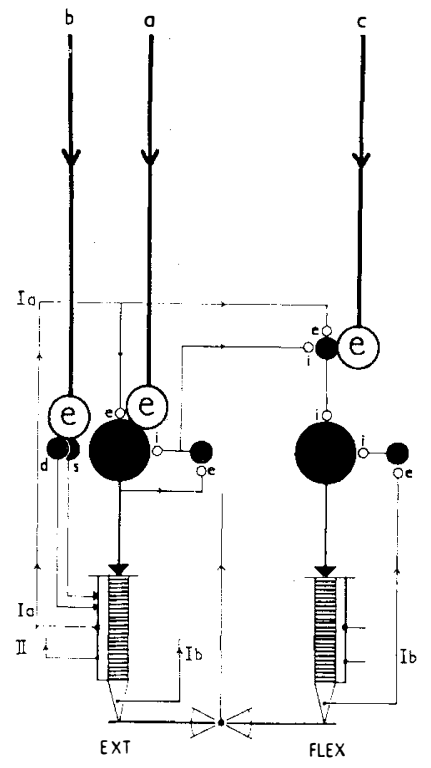

FIG. 8 which maintains extensor rigidity, which is abolished by cutting the ventral quadrants.

Effects of Stimulation of Descending Pathways on Reflex Arcs. The effects of stimulating the vestibulospinal system are summarized in Figure 8. This system makes monosynaptic connexions with extensor motoneurones (fig. 8, a), the quantity of

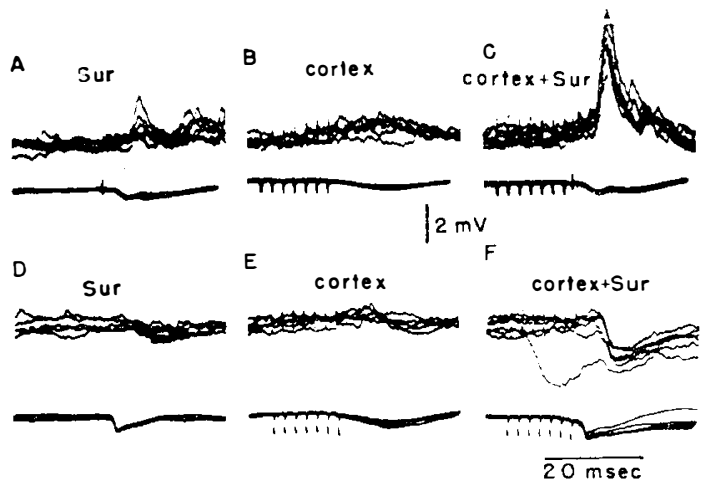

FIG. 9

Fig. 8.-Actions of the vestibulospinal tract, based on experiments of Lund and Pompeiano (I968) and Grillner (I969). See text.

Fig 9.-Excitatory convergence between cutaneous and corticospinal impulses at interneuronal level. See text. (Lundberg, I964.)

monosynaptic action being about 25 per cent. of that of the Group Ia input to the same motoneurones. It also connects monosynaptically with the static fusimotor neurones of extensor groups (fig. 8, b), and with the interneurones that mediate reciprocal inhibition from extensor spindles to flexor motoneurones (fig. 8, c). Thus it not only drives the extensor reflexes in $\alpha \gamma$ linkage but also reinforces their reciprocal inhibition of flexor reflexes.

A reticulospinal pathway that passes through the dorsal longitudinal bundle has homologous monosynaptic effects on flexor motoneurones (Grillner \& Lund, I968) and static fusimotors (Grillner, 1969) and reinforces the reciprocal inhibition of extensors by monosynaptically exciting the inhibitory interneurones.

The cat's corticospinal pathway does not, as does the primate's, make monosynaptic connexions with motoneurones. It operates through the interneurones of flexor arcs, and probably also through interneurones specific to itself. Figure 9 illustrates some of the evidence. Figure 9, A-C are intracellular records from a flexor motoneurone. Weak stimulation of a cutaneous nerve (the sural) had almost no synaptic action on the membrane of this motoneurone (fig. 9, A). Neither did stimulation of the motor cortex (fig. 9, B). But when delivered together, the cutaneous input and the corticospinal input did evoke an EPSP (fig. 9, C). The interpretation of this result is that both inputs converged on common interneurores. By itself, the synaptic excitation produced by each was insufficient to bring the 
interneurones to their firing level. Together, they did fire the interneurones, and the interneuronal impulses synaptically excited the motoneurone.

A similar convergence of cutaneous and corticospinal excitation on interneurones mediating inhibition to an extensor motoneurone is illustrated in Figure 9, D-F. When the interneurones are fired by the combined inputs the motoneuron shows an IPSP.

If this interpretation is correct it should be possible to find the interneurones. Figure ro shows that this has been accomplished. It illus-

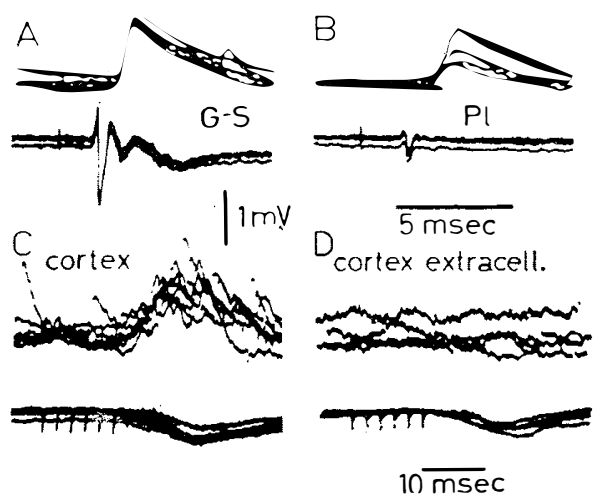

FIG. IO

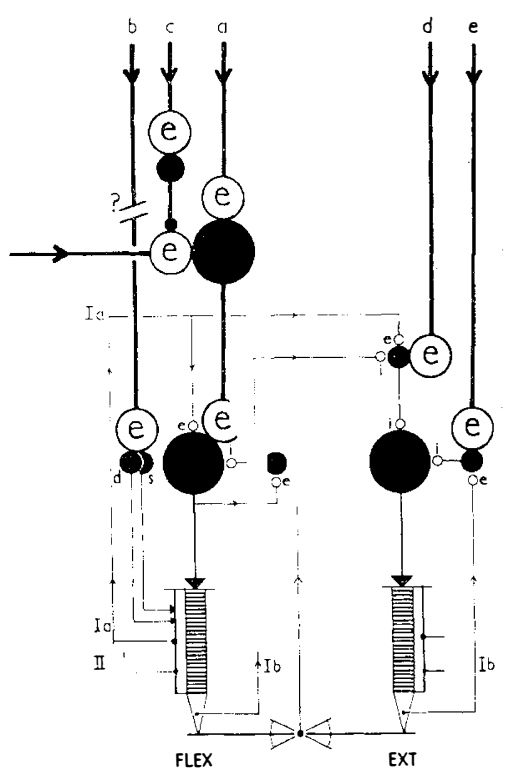

FIG. II

Fig. IO.-Monosynaptic convergence of peripheral and corticospinal impulses on an interneurone. See test. (Lundberg, 1964.)

Fig. I I.-Actions of the cat's corticospinal system. Note that the flexors are here drawn on the left of the joint. See text.

trates intracellular records from an interneurone which receives converging monosynaptic excitatory inputs from two peripheral nerves (fig. IO, A and B) and from the motor cortex (fig. IO, C).

The actions of the corticospinal system are summarised in Figure II. Note that in this Figure the flexors and extensors have been transposed to simplify the diagram. Figure II, A shows the action on flexor interneurones, already demonstrated in Figure 9, A-C and Figure IO. Figure I I, b shows the action on static and dynamic fusimotor neurones of flexors: the query signifies uncertainty as to whether or not it is monosynaptic. Figure I I, c shows presynaptic inhibition: of the input from the skin. Figure I I, d shows monosynaptic reinforcement of the reciprocal inhibition of extensor motoneurones from the primary endings of flexor spindles, and Figure I I, e the monosynaptic reinforcement of the inhibitory feedback from tendon organs.

Cutting the cat's pyramidal tract has remarkably little effect on behaviour. The clumsiness and weakness lasts for a few days at most. But although its gait becomes normal on a level floor, the cat remains permanently unable to pick its way accurately along the rungs of a horizontal ladder, and there is a measurable delay in the initiation of movement as compared with the limbs of the normal side. 
The actions of the rubrospinal system resemble very closely those of the corticospinal system.

These examples have been chosen to illustrate the power and precision of the experimental approach and its conceptual framework, and must not be taken to suggest that the pictures are already final, for fresh results are continually coming in.

The Actions of DOPA on the Cord of the Acute Spinal Cat. L3,4-dihydroxyphenylalanine (DOPA) is a precursor of noradrenaline (NA) which may well be a synaptic transmitter in the spinal cord. Unlike NA, DOPA penetrates the blood/ CNS barrier when injected intravenously. Synaptic terminals containing NA exist in the spinal grey matter, and survive for some time after transection of the cord. They are the terminations of fibres originating from cells in the reticular formation. Administration of DOPA may therefore, by providing material for synthesis of NA at these synapses, enable the physiologist to stimulate with the specific chemical transmitter at the terminals of an important noradrenergic reticulospinal system.

The action of this system, when thus thrown into action, is to block the shortlatency flexion that is normal in the acute spinal cat, and to uncover a larger and more delayed flexion reflex. With this delayed reflex goes a large (presynaptic inhibitory) depolarisation of the primary afferent arborisations of the Group Ia input. The static fusimotor neurones, which are normally silent in the acute spinal cat, are restored to activity. Gentle manipulation of the hindlimbs elicits rhythmic stepping movements. It seems possible that further work with DOPA will throw much-needed light on the spinal nervous networks that are responsible for stereotyped rhythmic activities such as stepping and scratching.

\section{CONCLUSION}

This article has tried to review the methods and some of the results of recent experiments on the cat's spinal cord and to give some idea of the conceptual developments which have been based upon them. Enough evidence has been presented to show how the brain could operate selectively and simultaneously on different reflex arcs, exciting some, inhibiting others. Spinal transection has specific effects on reflexes, releasing some and leaving others in a state of 'shock'. Such a concept is preferable to that of 'spinal shock' which implies a single, indivisible entity and tends to discourage curiosity about identifiable features in a more complex reality. There is imbalance rather than an equal depression of all functions. Even in acutely spinal man, when no reflexes can be elicited, some are likely to be less depressed than others. Detailed knowledge could well lead to practical consequences. Much might be done by deliberately avoiding stimulation of the receptive fields of 'unwanted' reflexes and by assiduous stimulation of the receptive fields of those whose early return (extensors, bladder, rectum) the physician wishes to encourage.

\section{REFERENCES}

\section{A. General}

The following books will be helpful to those who wish to pursue the subject in greater depth. EcCles, J. C. (1964). The Physiology of Synapses. Heidelberg: Springer-Verlag. Granit, R. (I966a). Charles Scott Sherrington: An appraisal. London: Nelson. Granit, R. (ed.) (I966b). Muscular Afferents and Motor Control. Stockholm: Almqvist \& Wiksell. 


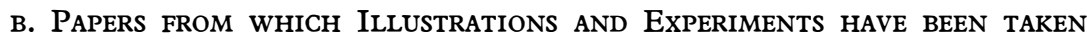

Grillner, S. (I969). Supraspinal and segment control of static and dynamic $\gamma$ motoneurones in the cat. Acta physiol. scand. Suppl. 327, p. I6.

GRILLNER, S. \& LUND, S. (I968). The origin of a descending pathway with monosynaptic action on flexor motoneurones. Acta physiol. scand., 74, 274-284.

HolmQvist, B. \& LUNDBERG, A. (I959). On the organization of the supraspinal inhibitory control of interneurons of various spinal reflex arcs. Arch. ital. Biol., 97, 340-356.

HolmQvist, B. \& LUNDBERG, A. (I96I). Differential supraspinal control of synaptic actions evoked by volleys in the flexion afferents in alpha motoneurones. Acta physiol. scand., 54. Suppl. I86.

Hultborn, H., Jankowska, E. \& Lindström, S. (1968). Recurrent inhibition from motor axon collaterals in interneurones monosynaptically activated from Ia afferents. Brain Research, 9, 367-369.

Lund, S. \& Pompeiano, O. (1968). Monosynaptic excitation of alpha motoneurones from supraspinal structures in the cat. Acta physiol. scand., 73, I-2I.

LUNDBERG, A. (1964). Supraspinal control of transmission in reflex paths to motoneurones and primary afferents. Progr. Brian Res., I2, 197-219.

\title{
SPINAL SHOCK AND REFLEX BEHAVIOUR IN MAN
}

\author{
By Professor Sir Ludwig Guttmann, C.B.E., M.D., F.R.C.P., F.R.C.S.
}

SINCE Marshall Hall (I84I) introduced the term 'spinal shock' for the state of in- or hypoexcitability of the isolated cord which exists immediately and in the early stages following transection of the spinal cord, this phenomenon has repeatedly been the subject of clinical and physiological research and discussion. While the majority of modern investigators came to the conclusion that spinal shock represents a dysbalance of the function of the internuncial neurons and not of the motoneurons, the intrinsic mechanism of this phenomenon and its variations amongst the various species in the evolutionary scale are still not wholly understood. One of the reasons for this is the fact that experimental workers in this field adopted different technical procedures and modes of investigations including different types of anaesthesia. This is in particular the case when the functions of the spinal cord have been studied in preparations of animals of higher types in the evolutionary scale. Koley's and Muckerjee's (1964) comments on the techniques used are as follows:

(I) Sherrington's preparations were not a spinal preparation, but a decapitate preparation.

(2) The technique of pithing the cord used by Goltz (1864) is suitable only in small animals such as frogs and guinea-pigs.

(3) Treat's technique (194I) used in cats by blind puncture and cauterisation, avoiding traumatic effects of laminectomy, has the uncertainty of complete separation of the cord.

(4) McDowall's technique, adopted more or less by Downman, McSwiney and Muckerjee himself is a preparation which converts the preparation into 'decerebrate with cordotomy'. Artificial respiration was always required to maintain the life of the animal.

Nevertheless, the modern concept of spinal shock is based on the work of Sherrington and his school, which suggests that the transient reflex depression in 